\title{
Nivel de estrés en niños(as) de primer año de primaria y correlación con alteraciones en su conducta
}

\author{
Stress level in children in their correlation \\ with behavior alterations
}

\footnotetext{
Est. Nydia Loredo Martínez* ${ }^{\star}$ Est. Diana Mejía Jiménez*, Est. Nancy Jiménez Bautista*, Mtra. Reyna Matus Miranda**

* Alumnas de la Licenciatura en Enfermería y Obstetricia ENEO-UNAM.

** Profesora de Carrera, Unidad de Investigación ENEO -UNAM.
}

\section{Resumen}

El trabajo escolar continuado bajo presión de tiempo y el ambiente físico son condiciones estresantes cuyos efectos provocan en los niños reacciones de adaptación. Los niños y adultos pueden mostrar diferentes modos de reaccionar frente al estrés; éste, juega un papel importante en las causas y el mantenimiento de problemas emocionales, lo que de acuerdo con ciertos autores es especialmente cierto en niños. El estrés puede ayudar en momentos de crisis ya que alerta los sentidos y hace estar en guardia, sin embargo también puede afectar la salud tanto física como mental, considerando lo anterior es importante que como enfermeras también valoremos este aspecto.

\section{Objetivo}

Identificar el nivel de estrés en niños(as) de primer año de primaria y valorar si existe correlación con alteraciones en su conducta.

\section{Metodología:}

Tipo de estudio observacional, correlacional, transversal, y descriptivo. Se integró un grupo de primer año de primaria de niños y niñas $(n=41)$, a los que se les aplicaron los instrumento con base en aceptación de sus padres. Se elaboraron y validaron dos cuestionarios, uno de 27 preguntas para medir el nivel de estrés, el segundo con
16 preguntas para valorar alteraciones en la conducta de los(as) niños(as). Se utilizaron las pruebas $U$ de Mann Whitney para comparar el nivel de estrés de niños(as) y la de correlación de Spearman para estimar la correlación de las variables nivel de estrés y conducta.

\section{Resultados}

34 niños presentaron niveles medios y altos de estrés, también tuvieron alteraciones en su conducta; Se obtuvo una $\mathbf{r}^{\mathbf{s}}=0.73$, con un nivel de significancia de $0.1($ (.432) entre nivel de estrés y conducta. Los 7 indicadores utilizados para la variable conducta tuvieron valores significativos, sin embargo los más altos son la agresividad y la frustración.

\section{Conclusiones}

1.- Identificar los niveles de estrés en niños(as) permitira el establecimiento de estrategias para su regulación en niveles no dañinos para su salud.

2.- Como enfermeras, este análisis, debiera formar parte de la valoración que se hace regularmente a estos grupos. 


\section{Abstract}

Continuous school activities under time pressure and unfavorable ambiences are stressful conditions which provoke adaptation reactions in children (and adults) are important causes of emotional problems. Stress con be helpful during certain moments because it alerts our senses, however, most of the time, it has a negative effect in our health.

Objective: Identify stress level among children in their first year of elementary school, and also assess if this stress is correlated to their behavior alterations.

Methodology

Basic, observational, co rrelational and descriptive study with a sample of 41 boys and girls in their first year of elementary school. A 27 item instrument was designed to estimate the stress level, and a 16 item instrument was designed to assess behavior alterations. Mann Whitney U Test were used to assess the differences between boys and girls. Spearman correlations were obtained to assess the associations among stress and behavior variables.

Results 34 children showed levels of stress from medium to high, and also alterations in their behaviors. Spearman's rho was .73 and turned out to be significant the 7 conduct variable indicators were all significant, especially aggressiveness and frustration.

Conclusions:

1 Identifying stress levels in children will result in strategies of control.

2 As nurses, this analysis should take por y of children's regular assessments.

\section{INTRODUCCIÓN}

En el medio escolar existen una serie de factores determinantes de salud para los niños que en él se desarrollan. Bajo cierta mirada se considera que la escuela debe constituirse como un entorno que aporte experiencias saludables, tomando en cuenta para ello desde el medio físico que incluye el propio edificio, su orientación, sus condiciones acústicas, la iluminación, su estado de higiene. Los servicios que presta, como el comedor, la vigilancia, así como la prevención de accidentes o la detección de anomalías o posibles problemas de salud, lo anterior resulta ser trascendental, pues estos aspectos pueden influir en el desarrollo físico y psicosocial del niño en etapa escolar. ${ }^{1}$

Dentro de este contexto el trabajo y la valoración que realizan durante sus prácticas los estudiantes de enfermería, revisten vital importancia, uno de ellos es el que se refiere a la identificación de niveles de estrés en los niños, pues como se observará más adelante, estos pueden llegar a ser un factor que influya en su grado de desarrollo académico y social.

El estrés se refiere a cualquier exigencia que produce un estado de tensión o amenaza y que exige un cambio o adaptación. El estrés como respuesta es la alteración causada por un estímulo nocivo o factores estresantes. Hans Selye definió el estrés como una respuesta inespecífica del organismo a cualquier demanda surgida y también se dedicó a estudiar los problemas de la tensión y le llamó "síndrome de adaptación general (SAG) o síndrome de estrés"2. Para el caso de los niños, el estrés se experimenta de muchas formas y varía según su nivel de desarrollo y experiencias previas en la vida. ${ }^{3}$

En términos generales y con motivo de este estudio, el estrés es considerado una respuesta orgánica funcional - hasta cierto punto normal - que sin embargo cuando sobrepasa ciertos niveles, puede llegar a afectar de manera física, psicológica o social limitando incluso el aprendizaje.

Se ha observado que es cada vez más común en niños en edades tempranas, quienes entre otras consecuencias desarrollan enfermedades y/o cambios en su conducta llegando incluso a repercutir en su nivel de desempeño educacional. ${ }^{4}$

Para definir lo que es el estrés, se pueden tomar como referencia tres enfoques:

Estrés como estímulo: la monotonía, el aislamiento, el trabajo continuado bajo presión de tiempo, el calor, el cambio abrupto de ambiente; son condiciones estresantes cuyo efectos generalmente provocan en todas las personas (en este caso en los niños) reacciones de adaptación; es importante comentar que, estas reacciones también pueden no ser adaptativas. Desde este punto de vista el nivel de estrés depende de las características del medio ambiente el cual de alguna manera exige conductas de adaptación. Se pueden identificar estresores externos que tienen que ver con el ambiente físico o social y estresores internos que son aquellos que tienen que ver con impulsos y deseos del propio sujeto.

Estrés como respuesta: aquí el niño muestra conductas como respuestas que intentan adaptarlo al estresor. 
Existen diferentes estilos de respuesta frente al estrés; reacciones que significan el uso de determinados mecanismos de defensa, inclusive desde la edad escolar. Considerando el aspecto fisiológico, Selye denominó Síndrome de adaptación general a la reacción fisiológica generalizada ante la experiencia de estrés. ${ }^{5}$

Estrés como amenaza percibida: desde otro punto se asume que el estrés proviene de la percepción y evaluación que la persona hace de la situación identificándola como evento amenazante para su propia seguridad. Aquí el sujeto evalúa y compara sus propias capacidades para hacer frente al estrés y la intensidad del estresor experimentando efectos fisiológicos y psicológicos consecuentes de esa evaluación.

En términos generales el estrés tiene un efecto directo sobre nuestro ser físico, así por ejemplo; lo que nos sucede cuando vivimos con fuertes sentimientos de disgusto, enojo o molestia y tendemos a encerrarnos en nosotros mismos, estar a la defensiva y a veces esto nos produce dolores de cabeza, estómago, malestar general etc. El estrés continuo ha probado causar un daño gradual en el sistema circulatorio el aparato digestivo, los pulmones los músculos y/o articulaciones, esto es aplicable tanto en niños como adultos. ${ }^{6}$

El lado positivo del estrés es que puede ayudarnos en momentos de crisis, ya que alerta los sentidos y nos hace estar en guardia para resolverlos, sin embargo como ya se comentó, también en grados extremos puede afectar la salud tanto física como mental.

\section{FUENTES DE ESTRÉS EN NIÑOS}

En el caso particular de los niños, ${ }^{7}$ el estrés puede estar relacionado con situaciones o experiencias vividas, entre las que se encuentran en primer lugar las relacionadas con su contexto escolar; el trabajo escolar o situaciones relacionadas con éste; cambio de salón, de escuela o de grupo o grado e incluso llegar tarde a las actividades escolares. Las interacciones sociales en este espacio académico; peleas o enojos entre compañeros, ridiculización y/o maltrato por parte de los profesores etc. Y lo que se refiere al trato de los profesores; particularmente cuando existen regaños o llamadas de atención o bien otras medidas disciplinarias.

La fuente de estrés en niños que ocupa el segundo lugar, se relaciona con su ambiente familiar; la pérdida de algún familiar directo, padre, madre, abuelo(a), hermano(a) etc, peleas o separación de la familia, integración de un nuevo miembro a este grupo social (hermanos, bebés, pareja de alguno de los padres etc).

Otras fuentes de estrés en los niños pueden ser las actividades extraescolares (deportes o de descanso) o incluso actividades consideradas como placenteras; fiestas, celebraciones (navidad, día de reyes, paseos etc).

Un hecho singular en el caso de los niños, es que el estrés puede estar relacionado con situaciones imaginarias o no vividas; aventuras, sueños o pesadillas (particularmente las que tienen que ver con seres monstruosos) etc.

Síntomas físicos y emocionales o de conducta que pueden presentar los niños derivados de algún nivel de estrés. $^{8}$

\section{Síntomas físicos}

- Dolor de cabeza

- Molestia estomacal o dolor estomacal vago (inespecífico)

- Problemas para dormir

- Pesadillas

- Mojar la cama, por primera vez o recurrentemente

- Disminución del apetito, cambios en los hábitos alimentarios

- Otros síntomas físicos sin enfermedad manifiesta.

\section{Síntomas emocionales o alteraciones de conducta}

- Ansiedad

- Preocupaciones

- Incapacidad de relajarse

- Miedos nuevos o recurrentes (miedo a la oscuridad, a estar solo o a los extraños)

- Aferrarse al adulto (padre, madre, abuelitos etc.), incapaz de perderlo de vista

- Puede o no hacer preguntas de manera reiterativa

- Rabia

- Llanto

- Gimoteo

- Incapacidad para controlar sus emociones

- Comportamiento agresivo

- Comportamiento terco

- Regresión a comportamientos típicos de etapas anteriores del desarrollo

- Reación al participar en actividades familiares o escolares.

- Cómo se manifiesta el Estrés

- Conductas regresivas: chuparse el dedo, mojar la cama, comerse las uñas, etc. 
- Retirada social poco característica del niño: no querer hablar con nadie, y parecer deprimido.

- Perdida de motivación o de capacidad de concentrarse en tareas.

- Cambios importantes en su conducta habitual.

- Perdida del apetito o del sueño.

- Irritabilidad sin explicación.

- Quejas físicas: dolor de cabeza, de estómago, malestar inexplicable etc.

- Problemas con los amigos y compañeros de clase.

\section{Relación estrés y conducta en niños}

Los niños pueden mostrar ciertos patrones en sus reacciones frente a los estresores, éstos son intentos adaptativos para ajustarse a las demandas del ambiente estresante, imaginémonos por un momento una línea recta; en un extremo están las conductas adaptativas y efectivas $y$ en el otro, los comportamientos desadaptativos frente a los estresores, entonces, las conductas que adopta el niño para enfrentar el estrés pueden ser vistas en estos extremos incluyendo los puntos intermedios.

Los cuatro patrones de respuesta al estrés pueden ser descritos de la siguiente manera ${ }^{9}$ :

- Respuesta Dependiente: falta de autoconfianza, dificultad para aceptar las críticas, pobre asertividad, poca participación en actividades.

- Respuesta Reprimida: mucha sensibilidad, fácilmente se molestan o se les hieren sus sentimientos, temerosos ante nuevas situaciones, poca confianza en sí mismos, preocupados innecesariamente.

- Respuesta Pasivo-Agresiva: frecuentemente son niños de bajo rendimiento académico, tienden a postergar sus deberes; poco cooperativos, despistados; sus notas tienden a bajar.

- Respuesta Impulsiva: exigente, desafiante, de temperamento explosivo; causarán incomodidad o molestia en sus interacciones con otros niños; Por otro lado puede ser muy activos pero descuidados en su trabajo escolar.

Desde este punto de vista, la conducta del niño bajo estrés puede ir desde el extremo pasivo al extremo activo; y por otro lado, del extremo introvertido al extremo extrovertido. Mientras más extremas sean las conductas (hacia los polos activo - pasivo o introversión - extroversión), más desadaptativo será el ajuste del niño a su ambiente.
Considerando todo lo ya señalado, el objetivo de este estudio fue Identificar el nivel de estrés en niños(as) de primer año de primaria y valorar si existía asociación de éste con alteraciones en su conducta.

\section{METODOLOGÍA}

Con un diseño no experimental, se llevó a cabo un estudio observacional, correlacional, transversal y descriptivo.

La población fueron 108 niños y niñas de primer grado de una Escuela Primaria del Distrito Federal. La muestra se constituyó por el 38\% de la población (41 sujetos; 22 niños y 19 niñas) cuyos padres y autoridades escolares aceptaron que dieran respuesta a los instrumentos una vez dados a conocer los objetivos del estudio (consentimiento informado), el tipo de muestreo fue no aleatorio de conveniencia.

Para el presente estudio, se analizaron las siguientes variables:

Nivel de estrés de los niños y niñas; grado de estrés que experimenta cada niño(a) en el momento en que se aplicó el instrumento, podía ir de 0 a 54 puntos.

Indicadores; Niveles de estrés - normal; de 1 a 17 puntos, medio; 18 a 36 puntos y alto; de 37 a 54 puntos.

Alteración en la conducta del (la) niño(a): son las diferentes formas en que los niños se relacionan y las actitudes de su comportamiento que se producen frente situaciones determinadas que le generan estrés, los indicadores considerados fueron:

Inquietud: estado de comportamiento que el (la) niño(a) presenta cuando le resulta difícil fijar su atención en algo.

Tristeza: falta de alegría en el (la) niño(a) al realizar sus actividades.

Ansiedad: estado en el que el (la) niño(a) se encuentra agitado(a) e inquieto(a).

Frustración: sentimiento de enojo por no poder realizar alguna actividad (de manera voluntaria o impuesta).

Agresividad: comportamiento de agresión física, verbal o psicológica del (la) niño(a) frente a situaciones de intolerancia hacia sus compañeros. 
Conflicto: situación difícil de resolver para un(a) niño(a) que se traduce en indecisión de su parte para adoptar una $u$ otra actitud o comportamiento.

Miedo: sentimiento negativo que hacen que el (la) niño(a) tenga temor sobre algunos aspectos de su vida.

\section{Instrumentos de medición}

Partiendo de las variables e indicadores considerados, se procedió a la elaboración de dos instrumentos: el primero para medir el nivel de estrés cuya primera versión constaba de 57 preguntas, el segundo para valorar alteraciones en la conducta derivadas del nivel de estrés de 33 preguntas.

Ambos fueron validados por profesionales expertos en el tema; una Psiquiatra infantil, dos Psicólogos educativos, una Trabajadora Social y una Maestra de Educación Primaria, quienes opinaron respecto a las variables e indicadores considerados, el tipo y número de preguntas, las escalas de respuestas y emitieron sugerencias para el trabajo que se debía hacer con los alumnos previo a la aplicación de los instrumentos, derivado de estas observaciones los instrumentos quedaron de la siguiente manera:

El primer instrumento para medir el nivel de estrés se constituyó de 27 preguntas y el segundo instrumento para valorar alteraciones en la conducta integrado por 16 preguntas. Ambos con opciones de respuestas de Sí, a veces y No las cuáles iban acompañadas de una carita de la siguiente manera:

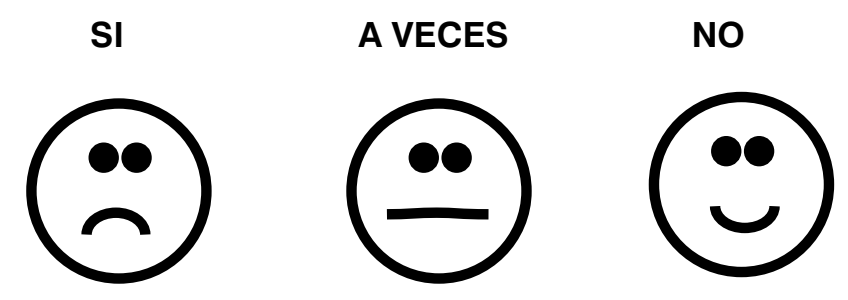

Se procuró que las preguntas quedaran estructuradas de manera simple y especifica con un lenguaje claro y conciso para el mejor entendimiento de los niños y niñas de acuerdo a cada indicador.

La prueba piloto fue aplicada a 10 niños dentro de un rango de edad de 6 a 7 años correspondiente a primer año de primaria (en un centro escolar ajeno al estudio), esta actividad permitió el ajuste al lenguaje utilizado en los cuestionarios, estimación del tiempo en que los niños dieron respuesta a los mismos y la eliminación de términos que aun no comprendían así como algunos pequeños ajustes en la hoja de respuestas. Es importante comentar que estos diez pequeños no fueron incluidos en el análisis de los datos.

La aplicación final del primer instrumento (para valorar el nivel de estrés) fue el día, la hora y el lugar asignado por las autoridades de la Escuela, en un mismo momento a los 41 alumnos, a los cuáles se les dio la explicación de manera breve pero precisa. Acorde a la sugerencia de los jueces expertos, el instrumento se dividió en dos partes para su aplicación y en el intermedio entre una y otra de las partes se realizó con los niños ciertos ejercicios para su concentración.

A partir de los puntajes obtenidos en este instrumento, se seleccionó a los niños que tuvieron nivel de estrés medio y alto (37 niños), a éstos se les aplicó el segundo instrumento para valorar alteraciones en su conducta. Esta segunda medición se efectúo ese mismo día, posterior a que los pequeños tuvieron un periodo de descanso y tomaron un pequeño refrigerio.

En ambos instrumentos se utilizó una escala de medición ordinal; se otorgaron valores de respuesta según el tipo de enunciado; para los planteados en sentido favorable se les asignó una puntuación de 0,1 y 2, y para los desfavorables 2,1 y 0 respectivamente, posteriormente se sumaron todos los puntos obtenidos.

Para el instrumento para determinar nivel de estrés, dependiendo del puntaje obtenido se les ubicó en los siguientes niveles:1 a 17 puntos = nivel normal de estrés, de 18 a 36 puntos $=$ nivel medio de estrés y de 37 a 54 puntos $=$ nivel alto de estrés

Para el caso del cuestionario para valorar alteraciones en la conducta los rangos de puntaje fueron: de 1 a 16 puntos $=$ conducta alterada de manera moderada y de 17 a 32 puntos = conducta alterada de mayor manera

Tomando en consideración que se utilizó una escala de medición ordinal en ambos instrumentos, se utilizó estadística descriptiva e inferencial con las siguientes pruebas estadísticas No Paramétricas; U de Mann Whitney para comparar los niveles de estrés del grupo de las niñas vs los niños; la prueba de correlación de Spearman para determinar la intensidad de asociación entre la variable de nivel de estrés y conducta y la prueba de $\mathrm{Chi}^{2}$ se utilizó para establecer cual de los indicadores de conducta era el más alterado.

Es importante comentar que la realización de este estudio estuvo apegada a la Declaración de Helsinki de la Asociación Médica Mundial particularmente en los Princi- 
pios Éticos para la Investigación Médica en Niños ${ }^{10}$ en lo referente a consentimiento, privacidad y fines de la investigación en niños.

\section{RESULTADOS}

Para identificar el nivel de estrés, se aplicó el primer instrumento a 22 niños y 19 niñas de los 108 de la población blanco (los 67 niños restantes no cumplieron con el criterio de inclusión de que los familiares aceptaran que participaran en el estudio).

Después de esta aplicación, en el nivel normal de estrés se situaron 7 niños, en el nivel medio fueron 33 niños y sólo 1 se ubicó en un nivel alto de estrés.

Al hacer un subgrupo de análisis por sexo de estos 34 casos que se determinaron en niveles medio y alto de estrés; se observó que 17 niños se ubicaron en el nivel 2 de estrés (medio) y un caso para nivel 3 (Alto). Respecto a las niñas las 16 integrantes de este subgrupo se concentraron en nivel 2 de estrés (medio). Al hacer una comparación de estos grupos por sexo, a través de la prueba de U de Mann - Whitney se observan diferencias estadísticamente significativas entre ambos grupos (tabla1)

\section{Tabla 1. Comparación de medianas globales de niveles de estrés por género}

\begin{tabular}{cc} 
Sexo & Mediana \\
Niños & 26 \\
$\mathrm{n}=18$ & $(17$ a 41$)$ \\
\hline Niñas & 29.5 \\
$\mathrm{n}=16$ & $(21$ a 35$)$ \\
\hline$p^{*}$ & .0003 \\
\hline$p^{*}$ Prueba $U$ de Mann-Whitney & \\
\hline
\end{tabular}

Una vez aplicado y calificado el segundo instrumento a este subgrupo de 34 niños y niñas - para determinar alteraciones en la conducta - se observó que entre
Tabla 2. Comparación de medianas globales de conducta por género

\begin{tabular}{cc} 
Género & Mediana \\
Niños & 20 \\
$\qquad n=18$ & $(18$ a 34) \\
\hline Niñas & \\
\hline$n=16$ & $(17$ a 28) \\
\hline$p^{*}$ & .0001 \\
\hline$p^{*}$ Prueba u de Mann-Whitney & \\
\hline
\end{tabular}

ambos sexos, las medianas globales de conducta son semejantes, sin embargo también con la prueba $U$ de Mann - Whitney se observan diferencias estadísticamente significativas a favor de los niños, la explicación a estas diferencias se encuentra en los rangos de calificaciones mínimas y máximas de ambos grupos (tabla 2).

Al buscar la intensidad de asociación entre la variable nivel de estrés y alteración de la de conducta, a través de la prueba de correlación de Sperman, se obtuvo una $r s=0.73$, con un nivel de significancia de 0.1 (.432), por lo tanto puede considerarse que existe correlación tendiente a lo alto entre el nivel de estrés y las alteraciones en la conducta encontradas en los 34 niños y niñas; con una $p<0.01$ (gráfica 1).

Algo interesante es cuando se determinó el nivel de estrés; los niños mostraron puntajes heterogéneos entre sí, con un rango de 17 a 41 puntos. En el caso de las niñas los puntajes fueron de 21 a 35 siendo más homogéneos de niña a niña, por lo que se deduce que las situaciones estresantes que perciben los niños y las niñas son similares pero lo manejan y demuestran de distinta forma.

Para conocer cual de los indicadores de conducta era el más alterado, se utilizó la prueba de $\mathrm{Chi}^{2}$, con ésta se determinó que los 7 indicadores considerados tuvieron valores significativos, sin embargo los que tiene más alta significancia son la agresividad con una $\mathrm{Chi}^{2}$ calculada de 979.15 y la frustración con una Chi $^{2}$ calculada de 649.26 ambas con un grado de confianza de 0.01, (cuadro 1). 


\section{Cuadro 1 \\ Comparación por indicadores \\ de la Variable alteración de la conducta}

\begin{tabular}{|l|l|c|c|}
\hline \multicolumn{1}{|c|}{ Indicador } & \multicolumn{1}{|c|}{ Chi2 } & \multicolumn{1}{|c|}{$\begin{array}{c}\text { Grados } \\
\text { de } \\
\text { libertad }\end{array}$} & $\begin{array}{c}\text { Nivel de } \\
\text { confianza 0.01 }\end{array}$ \\
\hline Miedo & 87.82 & 33 & $>50.892$ \\
\hline Conflicto & 97.24 & 33 & $>50.893$ \\
\hline Tristeza & 197.78 & 33 & $>50.894$ \\
\hline Inquietud & 379.58 & 33 & $>50.895$ \\
\hline Ansiedad & 441.64 & 33 & $>50.896$ \\
\hline Frustración & 649.26 & 33 & $>50.897$ \\
\hline Agresividad & 979.15 & 33 & $>50.898$ \\
\hline
\end{tabular}

\section{DISCUSIÓN}

Uno de los primeros aspectos en los que queremos reflexionar se refiere a la selección del tema de estrés infantil como motivo de estudio; considerando las fuentes de información consultadas, este es un aspecto que resulta de vital importancia sobre la salud de los pequeños,la literatura refiere que el estrés puede influir tanto positiva como negativamente, sin embargo cuanto más pequeño el niño, más impacto tienen los eventos nuevos, y más poderoso y posiblemente negativo llega a ser el estrés, así, cierta cantidad de estrés forma una parte normal de la vida cotidiana de un niño e incluso una influencia positiva; no obstante, el estrés excesivo puede tener efectos tanto inmediatos como de largo plazo en la adaptabilidad de los niños a situaciones nuevas ${ }^{11}$, incluso de acuerdo con ciertos autores, la reacción de estrés en el primer año escolar puede suponer un factor de riesgo de aparición de problemas posteriormente.12

Considerando lo anterior resaltamos la importancia de detectar a tiempo el nivel de estrés en los(as) niños(as) por que de esta manera se podrían evitar problemas de adaptación social y enfermedades en cualquier etapa posterior de su desarrollo, pues existen estudios donde se observa que la edad de los niños es un factor determinante para su respuesta a al estrés. . $^{13,14}$

Los instrumentos para medir el nivel de estrés en niños y el grado de alteración de la conducta, nos parece que

Gráfica 1

rs $=0.73$, con un nivel de significancia de 0.1 (.432). Prueba de Spearman

Correlación global de Niveles de estrés y conducta

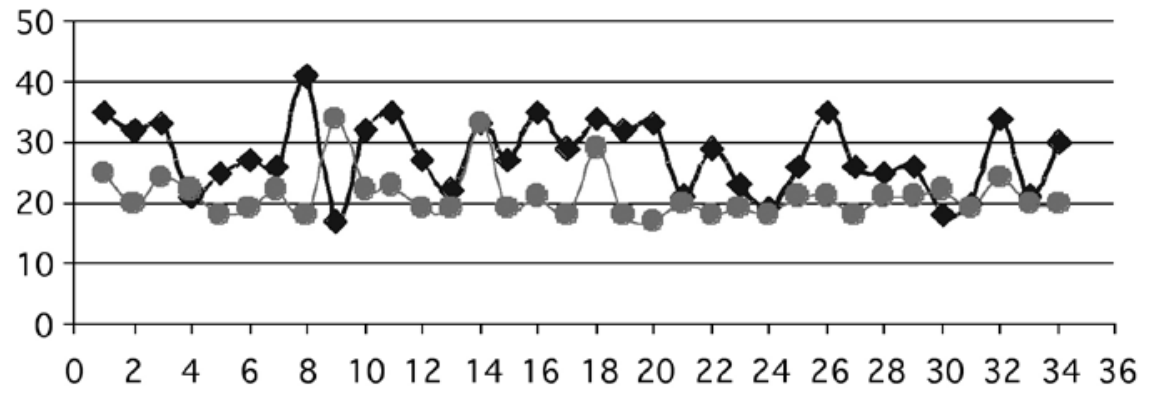

No. de caso

$\multimap$ Nivel de estrés $-\bullet$ Conducta 
en términos generales lograron su cometido de permitir establecer las diferencias en ambas variables en los niños y las niñas, lo cual para las enfermeras es primordial conocer y detectar a tiempo posibles alteraciones que a la larga puedan tener repercusiones en la salud y en la adaptabilidad social de esta población.

No podemos dejar de reconocer que una de las principales limitaciones de este estudio es su carácter transversal y correlacional (no de causalidad), porque se pueden presentar cambios en el estrés y conducta dependiendo de la situación particular de cada pequeño(a) así como en que momento de su desarrollo mental y físico se encuentren, por lo que también se tendrían que observar las posibles variables causales que estén interviniendo en los grados de estrés presentes en los niños y niñas lo que puede ser la base para futuros estudios relacionados con este tema.

Por otro lado el que se haya trabajado con una muestra no aleatoria pudiera parecer una debilidad del estudio, sin embargo el hecho que padres de familia y autoridades escolares hayan autorizado para trabajar con los pequeños nos permitió explorar de manera incipiente esta temática, para ir abriendo horizontes que nos permitan profundizar.

Considerando lo anterior, es deseable realizar de manera programada dentro de las prácticas clínicas que habitualmente realizan los estudiantes de enfermería dentro de Escuelas primarias, una evaluación del grado de estrés en los niños, contribuyendo así a valorar su salud de manera más integral; lo que aunado con la posibilidad de establecer de manera conjunta con otros profesionales de la salud (psicólogos, médicos, trabajadoras sociales etc.) y los propios maestros(as) responsables de los grupos, programas de intervención que coadyuven a transitar y contender a estos pequeños esta etapa de su vida y las diversas situaciones a las que se enfrentan (y que les pueden generar estrés en niveles dañinos para su salud) de la mejor manera.

\section{CONCLUSIONES}

1. Dentro de la valoración que realizan los estudiantes de enfermería durante sus prácticas en ámbitos escolares, es importante determinar también el nivel de estrés en que se encuentran los(as) niños(as) a fin de establecer estrategias de manera conjunta para regularlo en niveles no dañinos para su salud.

2. Continuar con esta temática de análisis a fin de correlacionar nivel de estrés con situaciones o vivencias específicas de los(as) niños(as), incluso recurriendo a abordajes metodológicos de carácter cualitativo.

\section{REFERENCIAS BIBLIOGRÁFICAS}

1 Perea Q Mr: Curso educación para la Salud: Fundamentos y justificación de la educación para la salud. Madrid. UNED, 1997; 139.

2 Hans S.: El descubrimiento del estrés. http://hipatis.morelos. gob.mx/no4/el estres.htm. [consultado 16 de febrero de 2009.]

3 Jewett, J. et al. "El estrés y los niños pequeños" http://www. ericdigest.org/2003-4/pequenos.html.,2003, [Consultado: el 27 de septiembre de 2007]

4 Vega Valero Z. et al. Estrés y ambiente familiar en niños. En http://www.psicologiacientifica.com/bv/psicologia-409-6estres-y-ambiente-familiar-en-ninos.html [consultado julio 2009].

5. Selye H. El descubrimiento del estrés en http://hypatia.morelos.gob.mx/no4/el_estres.htm [consulta agosto 2009]

6 Faber Cerril F., et al. Control emocional y salud mental. Editorial Trillas 1991, p .25

7 Merino SC. Visión introductoria al estrés infantil. http://www. psicopedagogia.com/marcos.html?estres.html. [consulta julio 2009]

8 Estrés en la niñez, en: http://www.healthbasis.com/spanish\% 20health\%20ilustrated0encyclopedia/5/002059.htm, 2004, [Consulta: el 27 de septiembre de 2007]

9 Chandler LA. The Stress Response Escala for children. A mesure for behavioral maladjust ment. University of Pittsburgf. Citado por Merino SC. Visión introductoria al estrés infantil. http://www.psicopedagogia.com/marcos. html?estres.html. [consulta julio 2009].

10 "Declaración de Helsinki de la Asociación Médica Mundial", en www.wma.net/s/policy/pdf/17c.pdf "Ética para la investigación en niños" en www.cgcom.org/notas_prensa/2007/07_10_05_amm.html - 22k, [Consultado el 22 de noviembre de 2007]

11 Jewett $J$ Y Peterson $\mathrm{K}$. El estrés y los niños pequeños. http://www.ericdigests.org/2003-4/pequenos.html [consultado julio 2009].

12 Rende y Plomín, 1992 en Trianes, Ma. Victoria, "Niños con estrés" $2^{a}$ ed., Alfa omega, 2002, pág. 103

13 Jackson EL. Observaciones de comportamientos del estrés manifestados por niños en una clase de kindergarten. ECRP Vol.11 No.1 Primavera de 2009 http://ecrp.uiuc. edu/v11n1/jackson-sp.html [consultado julio 2009].

\section{DIRECCIÓN PARA CORRESPONDENCIA}

Loredo Martínez Nydia: nylu_moon@yahoo.com.mx Mejía Jiménez Diana: dana_597@yahoo.com.mx 